\author{
D. Serikbaev ${ }^{1,3, *}$, N. Tokmagambetov ${ }^{2,3}$ \\ ${ }^{1}$ Al-Farabi Kazakh National University, Almaty, Kazakhstan; \\ ${ }^{2}$ Institute of Mathematics and Mathematical Modeling, Almaty, Kazakhstan; \\ ${ }^{3}$ Ghent University, Ghent, Belgium \\ (E-mail: serykbaev.daurenbek@gmail.com,niyaz.tokmagambetov@ugent.be)
}

\title{
A source inverse problem for the pseudo-parabolic equation with the fractional Sturm-Liouville operator
}

\begin{abstract}
A class of inverse problems for restoring the right-hand side of the pseudo-parabolic equation with one fractional Sturm-Liouville operator is considered. In this paper, we prove the existence and uniqueness results of the solutions using by the variable separation method that is to say the Fourier method. We are especially interested in proving the existence and uniqueness of the solutions in the abstract setting of Hilbert spaces. The mentioned results are presented as well as for the Caputo time fractional pseudoparabolic equation. There are many cases in which practical needs lead to problems determining the coefficients or the right side of a differential equation from some available decision data. These are called inverse problems of mathematical physics. Inverse problems arise in various areas of human activity, such as seismology, mineral exploration, biology, medicine, industrial quality control goods, and so on. All these circumstances put the inverse problems among the important problems of modern mathematics.
\end{abstract}

Keywords: Pseudo-parabolic equation, inverse problem, fractional Sturm-Liouville operator, Caputo fractional derivative.

\section{Introduction}

In this paper we consider pseudo-parabolic equation generated by fractional Sturm-Liouville operator with Caputo time-fractional derivative. We investigate the equation

$$
\mathcal{D}_{t}^{\alpha}\left[u(t, x)+\partial_{+a, x}^{\alpha} D_{b-, x}^{\alpha} u(t, x)\right]+\partial_{+a, x}^{\alpha} D_{b-, x}^{\alpha} u(t, x)=f(x),
$$

for $(t, x) \in \Omega=\{(t, x) \mid 0<t \leq T<\infty, a \leq x \leq b\}$, where $\mathcal{D}_{t}^{\alpha}$ is the Caputo derivative and $\partial_{+a, x}^{\alpha} D_{b-, x}^{\alpha}$ is the fractional Sturm-Liouville operator which is defined in the next section.

In many physical problems, it is required to determine the coefficients or the right-hand side (the original term, in the case of the diffusion equation) in the differential equation from some available information; These problems are known as inverse problems. Similar problems are poorly formulated in the sense of Hadamard. A number of papers consider the problem of solvability of inverse problems for the equations of diffusion and anomalous diffusion (see [1-9] and references therein).

\section{Definitions of fractional operators}

We begin this paper with a brief introduction of several concepts that are important for the further studies.

Definition 1 [10]. The Riemann-Liouville fractional integral $I^{\alpha}$ of order $\alpha>0$ for an integrable function is defined by

$$
I^{\alpha}[f](t)=\frac{1}{\Gamma(\alpha)} \int_{c}^{t}(t-s)^{\alpha-1} f(s) d s, t \in[c, d],
$$

where $\Gamma$ denotes the Euler gamma function.

\footnotetext{
${ }^{*}$ Corresponding author.

E-mail: serykbaev.daurenbek@gmail.com
} 
Definition 2 [10]. The Riemann-Liouville fractional derivative $D^{\alpha}$ of order $\alpha \in(0,1)$ of a continuous function is defined by

$$
D^{\alpha}[f](t)=\frac{d}{d t} I^{\alpha}[f](t), t \in[c, d] .
$$

Definition 3 [10]. The Caputo fractional derivative of order $0<\alpha<1$ of a differentiable function is defined by

$$
\mathcal{D}_{t}^{\alpha}[f](t)=D^{\alpha}\left[f^{\prime}(t)\right], t \in[c, d] .
$$

Definition 4 [10]. Let $f \in L^{1}[a, b],-\infty \leq a<t<b \leq+\infty$ and $f * K_{m-\alpha}(t) \in W^{m, 1}[a, b]$, $m=[\alpha], \alpha>0$. The Caputo fractional derivative $\partial_{+a}^{\alpha}$ of order $\alpha \in \mathbb{R}(m-1<\alpha<m, m \in \mathbb{N})$ is defined as

$$
\partial_{+a}^{\alpha} f(t)=D_{+a}^{\alpha}\left[f(t)-f(a)-f^{\prime}(a) \frac{(t-a)}{1 !}-\ldots-f^{(m-1)}(a) \frac{(t-a)^{m-1}}{(m-1) !}\right] .
$$

If $f \in C^{m}[a, b]$ then, the Caputo fractional derivative $\partial_{+a}^{\alpha}$ of order $\alpha \in \mathbb{R}(m-1<\alpha<m, m \in \mathbb{N})$ is defined as

$$
\partial_{+a}^{\alpha}[f](t)=I_{+a}^{m-\alpha} f^{(m)}(t)=\frac{1}{\Gamma(m-\alpha)} \int_{a}^{t}(t-s)^{m-1-\alpha} f^{(m)}(s) d s .
$$

\section{Fractional Sturm-Liouville operator}

We study the operator generated by the integro-differential expression

$$
\mathcal{L}(u)=\partial_{+a}^{\alpha} D_{b-}^{\alpha} u, a<x<b,
$$

and the conditions

$$
I_{b-}^{1-\alpha} u(a)=0, I_{b-}^{1-\alpha} u(b)=0
$$

where $\partial_{+a}^{\alpha}$ is the left Caputo derivative of order $\alpha \in(0,1]$ of $u$,

$$
D_{b-}^{\alpha} u(x)=-\frac{1}{\Gamma(1-\alpha)} \frac{d}{d x} \int_{x}^{b}(\xi-x)^{-\alpha} u(\xi) d \xi
$$

is the right Riemann-Liouville derivative of order $\alpha \in(0,1]$ of $u$, and

$$
I_{b-}^{\alpha} u(x)=\frac{1}{\Gamma(\alpha)} \int_{x}^{b}(\xi-x)^{\alpha-1} u(\xi) d \xi
$$

is the right Riemann-Liouville integral of order $\alpha \in(0,1]$ of $u,[10]$. The fractional Sturm-Liouville operator (2)-(3) is self-adjoint and positive in $L^{2}(a, b)$ (see [11-14]). The spectrum of the fractional Sturm-Liouville operator generated by the equations (2)-(3) is discrete, positive and real valued, and the system of eigenfunctions is a complete orthogonal basis in $L^{2}(a, b)$.

So we can denote eigenvalues and eigenfunctions accordingly by $\lambda_{\xi}$ and $e_{\xi}(x)$. That say us for $e_{\xi}(x) \in L^{2}(a, b)$ following identity is hold:

$$
\mathcal{L} e_{\xi}(x)=\lambda_{\xi} e_{\xi}(x), \lambda_{\xi} \in \mathbb{R}_{+} .
$$

Where $\mathcal{I}$ is a countable set and $\forall \xi \in \mathcal{I}$.

\section{Formulation of the problem}


We aim to find a couple of functions $(u(t, x), f(x))$ satisfying the equation (1) with an initial condition

$$
u(0, x)=\varphi(x), x \in[a, b]
$$

and with an additional information

$$
u(T, x)=\psi(x), x \in[a, b] .
$$

By using $\mathcal{L}$-Fourier analysis we obtain existence and uniqueness results for this problem.

We say a solution of the problem (1), (5), (6) is a pair of functions $(u(t, x), f(x))$ such that they satisfy equation (1) and conditions (5), (6) where $u(t, x) \in C^{\alpha}\left([0, T], \mathcal{H}^{1}\right), 0<\alpha \leq 1$ and $f(x) \in L^{2}(a, b)$.

Now, to investigate our problem, we need to define the Hilbert space $\mathcal{H}^{1}$.

Definition 5. The Hilbert space $\mathcal{H}^{1}$ is defined by

$$
\mathcal{H}^{1}:=\left\{u \in L^{2}(a, b): \mathcal{L} u \in L^{2}(a, b)\right\} .
$$

\section{Main results}

For problem (1), (5), (6) the following theorem holds.

Theorem. Let $\varphi, \psi \in \mathcal{H}^{1}$. Then a solution $u(t, x) \in C^{\alpha}\left([0, T], \mathcal{H}^{1}\right), 0<\alpha \leq 1, f(x) \in L^{2}(a, b)$ of problem (1), (5), (6) exists, is unique, and can be written in the form

$$
\begin{gathered}
u(x, t)=\varphi(x)+\sum_{\xi \in \mathcal{I}} \frac{\left[\left(\partial_{+a, x}^{\alpha} D_{b-, x}^{\alpha} \psi, e_{\xi}\right)_{L^{2}(a, b)}-\left(\partial_{+a, x}^{\alpha} D_{b-, x}^{\alpha} \varphi, e_{\xi}\right)_{L^{2}(a, b)}\right]\left(1-E_{\alpha, 1}\left(-\frac{\lambda_{\xi}}{1+\lambda_{\xi}} t^{\alpha}\right)\right) e_{\xi}(x)}{\lambda_{\xi}\left(1-E_{\alpha, 1}\left(-\frac{\lambda_{\xi}}{1+\lambda_{\xi}} T^{\alpha}\right)\right)}, \\
f(x)=\partial_{+a, x}^{\alpha} D_{b-, x}^{\alpha} \varphi(x)+\sum_{\xi \in \mathcal{I}} \frac{\left[\left(\partial_{+a, x}^{\alpha} D_{b-, x}^{\alpha} \psi, e_{\xi}\right)_{L^{2}(a, b)}-\left(\partial_{+a, x}^{\alpha} D_{b-, x}^{\alpha} \varphi, e_{\xi}\right)_{L^{2}(a, b)}\right] e_{\xi}(x)}{1-E_{\alpha, 1}\left(-\frac{\lambda_{\xi}}{1+\lambda_{\xi}} T^{\alpha}\right)} .
\end{gathered}
$$

Where $E_{\alpha, \beta}$ is the Mittag-Leffler type function [15]:

$$
E_{\alpha, \beta}(z)=\sum_{m=0}^{\infty} \frac{z^{m}}{\Gamma(\alpha m+\beta)} .
$$

Proof. First of all, we start by proving an existence result. Let us look for functions $u(t, x)$ and $f(x)$ in the forms:

$$
u(t, x)=\sum_{\xi \in \mathcal{I}} u_{\xi}(t) e_{\xi}(x)
$$

and

$$
f(x)=\sum_{\xi \in \mathcal{I}} f_{\xi} e_{\xi}(x),
$$

where $u_{\xi}(t)$ and $f_{\xi}$ are unknown. Substituting (7) and (8) into problem (1), (5), (6) and using relationship (4) we obtain the following problem for the functions $u_{\xi}(t)$ and for the constants $f_{\xi}, \xi \in \mathcal{I}$ :

$$
\begin{gathered}
\mathcal{D}^{\alpha} u_{\xi}(t)+\frac{\lambda_{\xi}}{1+\lambda_{\xi}} u_{\xi}(t)=\frac{f_{\xi}}{1+\lambda_{\xi}}, \\
u_{\xi}(0)=\varphi_{\xi}, \\
u_{\xi}(T)=\psi_{\xi},
\end{gathered}
$$


where $\varphi_{\xi}, \psi_{\xi}$ are $\mathcal{L}$-Fourier coefficients of $\varphi(x)$ and $\psi(x)$ :

$$
\begin{aligned}
& \varphi_{\xi}=\left(\varphi, e_{\xi}\right)_{L^{2}(a, b)}, \\
& \psi_{\xi}=\left(\psi, e_{\xi}\right)_{L^{2}(a, b)} .
\end{aligned}
$$

General solution of the equation (9):

$$
u_{\xi}(t)=\frac{f_{\xi}}{\lambda_{\xi}}+C_{\xi} E_{\alpha, 1}\left(-\frac{\lambda_{\xi}}{1+\lambda_{\xi}} t^{\alpha}\right)
$$

where the constants $C_{\xi}, f_{\xi}$ are unknown. By using conditions (10) and (11), we can find they. We first find $C_{\xi}$ :

$$
\begin{gathered}
u_{\xi}(0)=\frac{f_{\xi}}{\lambda_{\xi}}+C_{\xi}=\varphi_{\xi} \\
u_{\xi}(T)=\frac{f_{\xi}}{\lambda_{\xi}}+C_{\xi} E_{\alpha, 1}\left(-\frac{\lambda_{\xi}}{1+\lambda_{\xi}} T^{\alpha}\right)=\psi_{\xi}, \\
\varphi_{\xi}-C_{\xi}+C_{\xi} E_{\alpha, 1}\left(-\frac{\lambda_{\xi}}{1+\lambda_{\xi}} T^{\alpha}\right)=\psi_{\xi} .
\end{gathered}
$$

Then

$$
C_{\xi}=\frac{\varphi_{\xi}-\psi_{\xi}}{1-E_{\alpha, 1}\left(-\frac{\lambda_{\xi}}{1+\lambda_{\xi}} T^{\alpha}\right)}
$$

$f_{\xi}$ is represented as

$$
f_{\xi}=\lambda_{\xi} \varphi_{\xi}-\lambda_{\xi} C_{\xi}
$$

Substituting $f_{\xi}, u_{\xi}(t)$ into formula $(7)$, we find

$$
u(x, t)=\varphi(x)+\sum_{\xi \in \mathcal{I}} C_{\xi}\left(E_{\alpha, 1}\left(-\frac{\lambda_{\xi}}{1+\lambda_{\xi}} t^{\alpha}\right)-1\right) e_{\xi}(x) .
$$

Using self-adjoint property of operator $\mathcal{L}$

$$
\left(\mathcal{L} \varphi, e_{\xi}\right)_{L^{2}(a, b)}=\left(\varphi, \mathcal{L} e_{\xi}\right)_{L^{2}(a, b)}
$$

and in respect that (4) we obtain

$$
\left(\varphi, e_{\xi}\right)_{L^{2}(a, b)}=\frac{\left(\mathcal{L} \varphi, e_{\xi}\right)_{L^{2}(a, b)}}{\lambda_{\xi}}
$$

and for $\psi(x)$ we can write analogously. Substituting these equality into formula of $C_{\xi}$ we can get that

$$
C_{\xi}=\frac{\left(\mathcal{L} \varphi, e_{\xi}\right)_{L^{2}(a, b)}-\left(\mathcal{L} \psi, e_{\xi}\right)_{L^{2}(a, b)}}{\lambda_{\xi}\left(1-E_{\alpha, 1}\left(-\frac{\lambda_{\xi}}{1+\lambda_{\xi}} T^{\alpha}\right)\right)}
$$

Putting this into the formula (12), we have

$$
u(t, x)=\varphi(x)+\sum_{\xi \in \mathcal{I}} \frac{\left[\left(\mathcal{L} \psi, e_{\xi}\right)_{L^{2}(a, b)}-\left(\mathcal{L} \varphi, e_{\xi}\right)_{L^{2}(a, b)}\right]\left(1-E_{\alpha, 1}\left(-\frac{\lambda_{\xi}}{1+\lambda_{\xi}} t^{\alpha}\right)\right) e_{\xi}(x)}{\lambda_{\xi}\left(1-E_{\alpha, 1}\left(-\frac{\lambda_{\xi}}{1+\lambda_{\xi}} T^{\alpha}\right)\right)},
$$


As the same way as (13), we obtain

$$
f(x)=\mathcal{L} \varphi(x)+\sum_{\xi \in \mathcal{I}} \frac{\left[\left(\mathcal{L} \psi, e_{\xi}\right)_{L^{2}(a, b)}-\left(\mathcal{L} \varphi, e_{\xi}\right)_{L^{2}(a, b)}\right] e_{\xi}(x)}{1-E_{\alpha, 1}\left(-\frac{\lambda_{\xi}}{1+\lambda_{\xi}} T^{\alpha}\right)} .
$$

The following Mittag-Leffler function's estimate is known by [16]:

$$
\left|E_{\alpha, \beta}(z)\right| \leq \frac{M}{1+|z|}, \arg (z)=\pi,|z| \rightarrow \infty .
$$

Now, we show that $u(t, x) \in C^{\alpha}\left([0, T], \mathcal{H}^{1}\right), f(x) \in L^{2}(a, b)$, that is

$$
\|u\|_{C^{\alpha}\left([0, T], \mathcal{H}^{1}\right)}=\max _{t \in[0, T]}\|u(t, \cdot)\|_{\mathcal{H}^{1}}+\max _{t \in[0, T]}\left\|\mathcal{D}_{t}^{\alpha} u(t, \cdot)\right\|_{\mathcal{H}^{1}}<\infty
$$

and

$$
\|f\|_{L^{2}(a, b)}<\infty
$$

Where

$$
\|u(t, \cdot)\|_{\mathcal{H}^{1}}=\|u(t, \cdot)\|_{L^{2}(a, b)}+\|\mathcal{L} u(t, \cdot)\|_{L^{2}(a, b)}
$$

and

$$
\left\|\mathcal{D}_{t}^{\alpha} u(t, \cdot)\right\|_{\mathcal{H}^{1}}=\left\|\mathcal{D}_{t}^{\alpha} u(t, \cdot)\right\|_{L^{2}(a, b)}+\left\|\mathcal{D}_{t}^{\alpha} \mathcal{L} u(t, \cdot)\right\|_{L^{2}(a, b)} \cdot
$$

Using by the estimate (15) we get following estimates for $u(t, x), \mathcal{L} u(t, x)$ and $\mathcal{D}_{t}^{\alpha} u(t, x)$ :

$$
\begin{aligned}
& \|u(t, x)\|_{C\left([0, T], L^{2}(a, b)\right)}^{2}=\| \varphi(x) \\
& +\sum_{\xi \in \mathcal{I}} \frac{\left[\left(\mathcal{L} \psi, e_{\xi}\right)_{L^{2}(a, b)}-\left(\mathcal{L} \varphi, e_{\xi}\right)_{L^{2}(a, b)}\right]\left(1-E_{\alpha, 1}\left(-\frac{\lambda_{\xi}}{1+\lambda_{\xi}} t^{\alpha}\right)\right) e_{\xi}(x)}{\lambda_{\xi}\left(1-E_{\alpha, 1}\left(-\frac{\lambda_{\xi}}{1+\lambda_{\xi}} T^{\alpha}\right)\right)} \|_{C\left([0, T], L^{2}(a, b)\right)}^{2} \\
& \lesssim\|\varphi\|_{L^{2}(a, b)}^{2}+\sum_{\xi \in \mathcal{I}} \max _{t \in[0, T]}\left|\frac{\left[\left(\mathcal{L} \psi, e_{\xi}\right)_{L^{2}(a, b)}-\left(\mathcal{L} \varphi, e_{\xi}\right)_{L^{2}(a, b)}\right]\left(1-E_{\alpha, 1}\left(-\frac{\lambda_{\xi}}{1+\lambda_{\xi}} t^{\alpha}\right)\right)}{\lambda_{\xi}\left(1-E_{\alpha, 1}\left(-\frac{\lambda_{\xi}}{1+\lambda_{\xi}} T^{\alpha}\right)\right)}\right|^{2}\left\|e_{\xi}\right\|_{L^{2}(a, b)}^{2} \\
& \lesssim\|\varphi\|_{L^{2}(a, b)}^{2}+\sum_{\xi \in \mathcal{I}}\left[\frac{\left|\left(\mathcal{L} \psi, e_{\xi}\right)_{L^{2}(a, b)}\right|^{2}+\left|\left(\mathcal{L} \varphi, e_{\xi}\right)_{L^{2}(a, b)}\right|^{2}}{\lambda_{\xi}^{2}}\right]<\infty \\
& \|\mathcal{L} u(x, t)\|_{C\left([0, T], L^{2}(a, b)\right)}^{2}=\| \mathcal{L} \varphi(x) \\
& +\sum_{\xi \in \mathcal{I}} \frac{\left[\left(\mathcal{L} \psi, e_{\xi}\right)_{L^{2}(a, b)}-\left(\mathcal{L} \varphi, e_{\xi}\right)_{L^{2}(a, b)}\right]\left(1-E_{\alpha, 1}\left(-\frac{\lambda_{\xi}}{1+\lambda_{\xi}} t^{\alpha}\right)\right) \mathcal{L} e_{\xi}(x)}{\lambda_{\xi}\left(1-E_{\alpha, 1}\left(-\frac{\lambda_{\xi}}{1+\lambda_{\xi}} T^{\alpha}\right)\right)} \|_{C\left([0, T], L^{2}(a, b)\right)}^{2} \\
& \lesssim\|\mathcal{L} \varphi\|_{L^{2}(a, b)}^{2}+\sum_{\xi \in \mathcal{I}} \max _{t \in[0, T]}\left|\frac{\left[\left(\mathcal{L} \psi, e_{\xi}\right)_{L^{2}(a, b)}-\left(\mathcal{L} \varphi, e_{\xi}\right)_{L^{2}(a, b)}\right]\left(1-E_{\alpha, 1}\left(-\frac{\lambda_{\xi}}{1+\lambda_{\xi}} t^{\alpha}\right)\right)}{\left(1-E_{\alpha, 1}\left(-\frac{\lambda_{\xi}}{1+\lambda_{\xi}} T^{\alpha}\right)\right)}\right|^{2}\left\|e_{\xi}\right\|_{L^{2}(a, b)}^{2} \\
& \lesssim\|\mathcal{L} \varphi\|_{L^{2}(a, b)}^{2}+\sum_{\xi \in \mathcal{I}}\left[\left|\left(\mathcal{L} \psi, e_{\xi}\right)_{L^{2}(a, b)}\right|^{2}+\left|\left(\mathcal{L} \varphi, e_{\xi}\right)_{L^{2}(a, b)}\right|^{2}\right]<\infty \\
& \left\|\mathcal{D}_{t}^{\alpha} u(x, t)\right\|_{C\left([0, T], L^{2}(a, b)\right)}^{2}
\end{aligned}
$$




$$
\begin{gathered}
=\left\|\sum_{\xi \in \mathcal{I}} \frac{\left[\left(\mathcal{L} \psi, e_{\xi}\right)_{L^{2}(a, b)}-\left(\mathcal{L} \varphi, e_{\xi}\right)_{L^{2}(a, b)}\right] D_{t}^{\alpha}\left(1-E_{\alpha, 1}\left(-\frac{\lambda_{\xi}}{1+\lambda_{\xi}} t^{\alpha}\right)\right) e_{\xi}(x)}{\lambda_{\xi}\left(1-E_{\alpha, 1}\left(-\frac{\lambda_{\xi}}{1+\lambda_{\xi}} T^{\alpha}\right)\right)}\right\|_{C\left([0, T], L^{2}(a, b)\right)}^{2} \\
\leq \sum_{\xi \in \mathcal{I}} \max _{t \in[0, T]} \mid \frac{\left[\left(\mathcal{L} \psi, e_{\xi}\right)_{L^{2}(a, b)}-\left(\mathcal{L} \varphi, e_{\xi}\right)_{L^{2}(a, b)}\right] E_{\alpha, 1}\left(-\frac{\lambda_{\xi}}{1+\lambda_{\xi}} t^{\alpha}\right)}{\left(1+\lambda_{\xi}\right)\left(1-E_{\alpha, 1}\left(-\frac{\lambda_{\xi}}{1+\lambda_{\xi}} T^{\alpha}\right)\right)}\left\|e_{\xi}\right\|_{L^{2}(a, b)}^{2} \\
\lesssim \sum_{\xi \in \mathcal{I}}\left[\frac{\left|\left(\mathcal{L} \psi, e_{\xi}\right)_{L^{2}(a, b)}\right|^{2}+\left|\left(\mathcal{L} \varphi, e_{\xi}\right)_{L^{2}(a, b)}\right|^{2}}{\left(1+\lambda_{\xi}\right)^{2}}\right]<\infty,
\end{gathered}
$$

and

$$
\begin{gathered}
\left\|\mathcal{D}_{t}^{\alpha} \mathcal{L} u(x, t)\right\|_{C\left([0, T], L^{2}(a, b)\right)}^{2} \\
=\left\|\sum_{\xi \in \mathcal{I}} \frac{\left[\left(\mathcal{L} \psi, e_{\xi}\right)_{L^{2}(a, b)}-\left(\mathcal{L} \varphi, e_{\xi}\right)_{L^{2}(a, b)}\right] D_{t}^{\alpha}\left(1-E_{\alpha, 1}\left(-\frac{\lambda_{\xi}}{1+\lambda_{\xi}} t^{\alpha}\right)\right) \mathcal{L} e_{\xi}(x)}{\lambda_{\xi}\left(1-E_{\alpha, 1}\left(-\frac{\lambda_{\xi}}{1+\lambda_{\xi}} T^{\alpha}\right)\right)}\right\|_{C\left([0, T], L^{2}(a, b)\right)}^{2} \\
\leq \sum_{\xi \in \mathcal{I}} \max _{t \in[0, T]}\left|\frac{\lambda_{\xi}\left[\left(\mathcal{L} \psi, e_{\xi}\right)_{L^{2}(a, b)}-\left(\mathcal{L} \varphi, e_{\xi}\right)_{L^{2}(a, b)}\right] E_{\alpha, 1}\left(-\frac{\lambda_{\xi}}{1+\lambda_{\xi}} t^{\alpha}\right)}{\left(1+\lambda_{\xi}\right)\left(1-E_{\alpha, 1}\left(-\frac{\lambda_{\xi}}{1+\lambda_{\xi}} T^{\alpha}\right)\right)}\right|^{2}\left\|e_{\xi}\right\|_{L^{2}(a, b)}^{2} \\
\lesssim \sum_{\xi \in \mathcal{I}}\left[\left|\left(\mathcal{L} \psi, e_{\xi}\right)_{L^{2}(a, b)}\right|^{2}+\left|\left(\mathcal{L} \varphi, e_{\xi}\right)_{L^{2}(a, b)}\right|^{2}\right] \\
+\sum_{\xi \in \mathcal{I}}\left[\frac{\left|\left(\mathcal{L} \psi, e_{\xi}\right)_{L^{2}(a, b)}\right|^{2}+\left|\left(\mathcal{L} \varphi, e_{\xi}\right)_{L^{2}(a, b)}\right|^{2}}{\left(1+\lambda_{\xi}\right)^{2}}\right]<\infty .
\end{gathered}
$$

Similarly for $f(x)$ we have the estimate

$$
\begin{aligned}
& \|f\|_{L^{2}(a, b)}^{2}=\left\|\mathcal{L} \varphi(x)+\sum_{\xi \in \mathcal{I}} \frac{\left[\left(\mathcal{L} \psi, e_{\xi}\right)_{L^{2}(a, b)}-\left(\mathcal{L} \varphi, e_{\xi}\right)_{L^{2}(a, b)}\right] e_{\xi}(x)}{1-E_{\alpha, 1}\left(-\frac{\lambda_{\xi}}{1+\lambda_{\xi}} T^{\alpha}\right)}\right\|_{L^{2}(a, b)}^{2} \\
& \quad \lesssim\|\mathcal{L} \varphi\|_{L^{2}(a, b)}^{2}+\sum_{\xi \in \mathcal{I}}\left|\frac{\left(\mathcal{L} \psi, e_{\xi}\right)_{L^{2}(a, b)}-\left(\mathcal{L} \varphi, e_{\xi}\right)_{L^{2}(a, b)}}{1-E_{\alpha, 1}\left(-\frac{\lambda_{\xi}}{1+\lambda_{\xi}} T^{\alpha}\right)}\right|\left\|e_{\xi}\right\|_{L^{2}(a, b)}^{2} \\
& \quad \lesssim\|\mathcal{L} \varphi\|_{L^{2}(a, b)}^{2}+\sum_{\xi \in \mathcal{I}}\left[\left|\left(\mathcal{L} \psi, e_{\xi}\right)_{L^{2}(a, b)}\right|^{2}+\left|\left(\mathcal{L} \varphi, e_{\xi}\right)_{L^{2}(a, b)}\right|^{2}\right]<\infty .
\end{aligned}
$$

Where, $L \lesssim Q$ denotes $L \leq C Q$ for some positive constant $C$ independent of $L$ and $Q$. Existence of the solution of problem (1), (5), (6) is proved.

Now, we start proving the uniqueness of the solution.

Let us suppose that $\left\{u_{1}(x, t), f_{1}(x)\right\}$ and $\left\{u_{2}(x, t), f_{2}(x)\right\}$ are solution of problem (1), (5), (6). Then $u(x, t)=u_{1}(x, t)-u_{2}(x, t)$ and $f(x)=f_{1}(x)-f_{2}(x)$ are solution of following problem:

$$
\begin{gathered}
\mathcal{D}_{t}^{\alpha}\left[u(x, t)+\partial_{+a, x}^{\alpha} D_{b-, x}^{\alpha} u(x, t)\right]+\partial_{+a, x}^{\alpha} D_{b-, x}^{\alpha} u(x, t)=f(x), \\
u(x, 0)=0,
\end{gathered}
$$




$$
u(x, T)=0 .
$$

By using (13) and (14) for (16)-(18) we easily see $u(x, t) \equiv 0, f(x) \equiv 0$. Uniqness of the solution of problem (1), (5), (6) is proved.

Discussion on further generalisations. Note that the results are derived here can be generalised by using the non-harmonic analysis developed in the papers $[17,18]$ with the general setting settled in $[19,20]$. Moreover, the reader is referred to [21-25] for interesting applications of the non-harmonic analysis to the different branches of partial differential equations.

\section{Acknowledgements}

This research was funded by the Science Committee of the Ministry of Education and Science of the Republic of Kazakhstan (Grant No. AP09259394).

\section{References}

1 Kaliev, I.A. \& Sabitova, M.M. (2010). Problems of determining the temperature and density of heat sources from the initial and final temperatures. J. Appl. Ind. Math., 4, 332-339. DOI: $10.1134 /$ S199047891003004X

2 Orazov, I. \& Sadybekov, M.A. (2012). One nonlocal problem of determination of the temperature and density of heat sources. Russ Math., 56, 60-64. DOI: 10.3103/S1066369X12020089

3 Orazov, I. \& Sadybekov, M.A. (2012). On a class of problems of determining the temperature and density of heat sources given initial and final temperature. Sib Math J, 53, 146-151. DOI: 10.1134/S0037446612010120

4 Torebek, B.T. \& Tapdigoglu, R. (2017). Some inverse problems for the nonlocal heat equation with Caputo fractional derivative. Mathematical Methods in the Applied Sciences, 40(18), 64686479. DOI: $10.1002 / \mathrm{mma} .4468$

5 Furati, K.M., Iyiola O.S. \& Kirane, M. (2014). An inverse problem for a generalized fractional diffusion. Applied Mathematics and Computation, Vol. 249, 24-31. DOI:10.1016/j.amc.2014.10. 046

6 Ismailov, M.I. \& Cicek M. (2016). Inverse source problem for a time-fractional diffusion equation with nonlocal boundary conditions. Applied Mathematical Modelling, 40(7), 4891-4899. DOI: 10.1016/j.apm.2015.12.020

7 Kirane, M. \& Malik A.S. (2011). Determination of an unknown source term and the temperature distribution for the linear heat equation involving fractional derivative in time. Applied Mathematics and Computation, 218(1), 163-170. DOI: 10.1016/j.amc.2011.05.084

8 Kirane, M., Samet, B. \& Torebek B.T. (2017). Determination of an unknown source term temperature distribution for the sub-diffusion equation at the initial and final data. Electronic Journal of Differential Equations, Vol. 2017, No. 257, 1-13.

9 Nguyen, H.T., Le, D.L. \& Nguyen, V.T. (2016). Regularized solution of an inverse source problem fora time fractional diffusion equation. Applied Mathematical Modelling, 40(19), 8244-8264.

10 Kilbas, A.A., Srivastava, H.M. \& Trujillo, J.J. (2006). Theory and Applications of Fractional Differential Equations. North-Holland: Mathematics Studies.

11 Tokmagambetov, N. \& Torebek, T.B. (2016). Fractional Analogue of Sturm-Liouville Operator. Documenta Math., 21, 1503-1514.

12 Tokmagambetov, N. \& Torebek, B.T. (2018). Green's formula for integro-differential operators. J.Math. Anal. Appl., 468(1), 473-479.

13 Tokmagambetov, N. \& Torebek, B.T. (2019). Fractional Sturm-Liouville Equations: Self-Adjoint Extensions. Complex Anal. Oper. Theory, 13, 2259-2267. DOI: 10.1007/s11785-018-0828-z 
14 Tokmagambetov, N. \& Torebek, B.T. (2018). Symmetric differential operators of fractional order and their extensions. Transactions of the Moscow Mathematical Society, 2018, 177-185.

15 Luchko, Y. \& Gorenflo, R. (1999). An operational method for solving fractional differential equations with the Caputo derivatives. Acta Math. Vietnam, 24, 207-233.

$16 \mathrm{Li}, \mathrm{Z}$., Liu, Y. \& Yamamoto, M. (2015). Initial-boundary value problems for multi-term timefractional diffusion equations with positive constant coefficients. Appl. Math. Comput., 257, 381397.

17 Delgado, J., Ruzhansky, M. \& Tokmagambetov, N. (2017). Schatten classes, nuclearity and nonharmonic analysis on compact manifolds with boundary. J. Math. Pures Appl., 107, 758-783.

18 Ruzhansky, M. \& Tokmagambetov, N. (2016). Nonharmonic analysis of boundary value problems. Int.Math. Res. Notices, 2016(12), 3548-3615.

19 Kanguzhin, B., Ruzhansky, M. \& Tokmagambetov, N. (2017). On convolutions in Hilbert spaces. Funct. Anal. Appl., 51(3), 221-224.

20 Ruzhansky, M. \& Tokmagambetov, N. (2018). Convolution, Fourier analysis, and distributions generated by Riesz bases. Monatsh. Math., 187(1), 147-170.

21 Ruzhansky, M. \& Tokmagambetov, N. (2017). Wave equation for operators with discrete spectrum and irregular propagation speed. Arch. Ration. Mech. Anal., 226(3), 1161-1207.

22 Ruzhansky, M. \& Tokmagambetov, N. (2018). On a very weak solution of the wave equation for a Hamiltonian in a singular electromagnetic field. Math. Notes, 103(5-6), 856-858.

23 Ruzhansky, M. \& Tokmagambetov, N. (2018). Nonlinear damped wave equations for the subLaplacian on the Heisenberg group and for Rockland operators on graded Lie groups. J. Differential Equations, 265(10), 5212-5236.

24 Muñoz, J.C., Ruzhansky, M. \& Tokmagambetov, N. (2019). Wave propagation with irregular dissipation and applications to acoustic problems and shallow waters. J. Math. Pures Appl.,123, 127-147.

25 Ruzhansky, M. \& Tokmagambetov, N. (2019). Wave Equation for 2D Landau Hamiltonian. Appl. Comput. Math., 18(1) 69-78.

Д. Серикбаев, Н. Токмагамбетов

\title{
Штурм-Лиувилль бөлшек туынды операторлы псевдопараболалық теңдеуі үшін қайнар көзді анықтаудың кері есебі
}

\begin{abstract}
Мақалада Штурм-Лиувилль бөлшек туынды операторлы псевдопараболалық теңдеудің оң жағын қалпына келтіру кері есептер класы қарастырылды. Авторлар айнымалыларды ажырату әдісін, яғни Фурье әдісін қолдана отырып, шешімнің бар және жалғыздығын дәлелдеді. Сонымен қатар абстракты Гильберт кеңістігінде шешімнің бар және жалғыздығы туралы нәтижелерді алды. Көрсетілген нәтижелер уақыт бойынша Капуто бөлшек туындылы псевдопараболалық теңдеу үшін алынды. Дифференциалдық теңдеудің шешімдеріне қатысты кейбір қосымша ақпараттар арқылы теңдеудің оң жағын анықтау немесе теңдеудің коэффициенттерін анықтау есептері практикалық жұмыстардан туындап отыр. Бұл математикалық физиканың кері есептері. Олар адам қызметінің әртүрлі салаларында пайда болады, мысалы, сейсмология, минералды барлау, биология, медицина, өнеркәсіптік сапаны бақылау өнімдері және т.б. Осы жағдайлардың барлығы қазіргі математиканың маңызды мәселелерінің қатарына кері есептер саласын енгізіп отыр.
\end{abstract}

Kiлm сөздер: псевдопараболалық теңдеу, кері есеп, бөлшек туынды Штурм-Лиувилль операторы, Капуто бөлшек туындысы. 
Д. Серикбаев, Н. Токмагамбетов

\title{
Обратная задача определения источника для псевдопараболического уравнения с дробным оператором Штурма-Лиувилля
}

\begin{abstract}
В статье рассмотрен класс обратных задач восстановления правой части псевдопараболического уравнения с дробным оператором Штурма-Лиувилля. Авторами доказаны результаты существования и единственности решений, с использованием метода разделения переменных, то есть методом Фурье. Кроме того, особенная заинтересованность наблюдается в доказательстве существования и единственности решений в абстрактной постановке гильбертовых пространств. Указанные результаты представлены для дробного псевдо-параболического уравнения Капуто по времени. Есть много случаев, в которых практические потребности приводят к задачам определения коэффициентов или правой части дифференциального уравнения по некоторым доступным данным решения. Это так называемые обратные задачи математической физики. Они возникают в различных областях человеческой деятельности, таких как сейсмология, разведка полезных ископаемых, биология, медицина, промышленные товары контроля качества и т.д. Все эти обстоятельства ставят обратные задачи в число важных проблем современной математики.
\end{abstract}

Ключевые слова: псевдопараболическое уравнение, обратная задача, дробный оператор ШтурмаЛиувилля, производная Капуто. 